\title{
Medical Needs Documented by Emergency Medical Services (EMS) Responders to Areas Affected by Typhoon Haiyan in the Philippines: Implications on Disaster Response Policy
}

\author{
Carlos Primero D. Gundran,, ${ }^{1,2}$ Hilton Y. Lam, ${ }^{2,3}$ Jaifred Christian F. Lopez, ${ }^{2,4}$ Emelia B. Santamaria, ${ }^{2}$ \\ Anna Cristina A. Tuazon ${ }^{2,5}$ and Lotgarda Tayao ${ }^{6}$ \\ ${ }^{1}$ Department of Health Policy and Administration, College of Public Health, University of the Philippines Manila \\ ${ }^{2}$ Health Emergencies and Disasters Study Group, National Institutes of Health, University of the Philippines Manila \\ ${ }^{3}$ Institute of Health Policy and Development Studies, National Institutes of Health, University of the Philippines Manila \\ ${ }^{4}$ Office of Research and Innovation, and College of Medicine, San Beda University, Manila \\ ${ }^{5}$ Department of Psychology, College of Social Sciences and Philosophy, University of the Philippines Diliman, Quezon City \\ ${ }^{6}$ Department of Medicine, Silliman University Medical Center, Dumaguete City
}

\begin{abstract}
Background. Despite existing disaster preparedness policies in the Philippines, there has not been any validated assessment of the quality of disaster medical response, which would require reliable aggregate data on patient diagnoses and management.
\end{abstract}

Objective. This mixed-methods study documented the diagnoses, triage classification and case management of patients seen by Philippine EMS groups who responded to the Typhoon Haiyan disaster in the Philippines in November and December 2013, as well as difficulties associated in gathering these data, using the Utstein-style Template for Uniform Data Reporting of Acute Medical Response in Disasters as framework.

Methods. Three hundred (300) individuals vetted by EMS organizations were invited to answer a survey modeled after the Utstein-style template, and submit tallies of patients seen. Out of 52 responses received, policy recommendations were subsequently generated on concerns assessed by the template using the nominal group technique.

Results. The submitted data yielded a total of 41,202 patients with information on age, sex, and diagnosis; 19,193 with triage classification; and 27,523 with information on case management. The focus group discussion underlined the absence of a standard communication and information management system. Participants recommended establishing such a system, and highlighted the role of the Department of Health - Health Emergency Management Bureau in coordinating disaster medical response efforts and information management.

Conclusion. This study underlines the importance of effective communication, and multisectoral coordination, to generate reliable data and thus, facilitate resource allocation for disaster medical response.

Key Words: cyclones, disaster medicine, emergency medical services, relief work, Typhoon Haiyan

\section{INTRODUCTION}

Corresponding author: Carlos Primero D. Gundran, MD, FPCEM, MScDM

Department of Health Policy and Administration

College of Public Health

University of the Philippines Manila

Taft Ave., Ermita, Manila 1000 Philippines.

Telephone: +6325232997

Email: carlosprimerogundran@yahoo.com
Typhoon Haiyan (Philippine name: Yolanda), one of the strongest typhoons ever recorded, made landfall on the Philippine island of Samar on 8 November 2013, with winds reaching a speed of $235 \mathrm{~km} / \mathrm{h}$, and producing gusts of up to $275 \mathrm{~km} / \mathrm{h}$ with a radius of up to $480 \mathrm{~km}$, thereby causing widespread devastation across the Visayas islands and northern Palawan. Moreover, according to the Philippine Atmospheric, Geophysical and Astronomical Services 
Administration report of 2013, the typhoon dumped severe rainfall, triggering storm surges and inundating coastal villages and low-lying towns.

The Philippine National Disaster Risk Reduction and Management Council (NDRMMC) reported that the typhoon left 6,300 people dead, 28,688 injured and 1,062 missing. In addition, a total of 3.42 million families in 648 cities and municipalities, were affected by the typhoon. Specifically, 93\% of casualties were recorded in Region 8, which includes the provinces of Leyte, Samar, and Eastern Samar. The NDRMMC reported that total damages caused by the typhoon amounted to approximately PHP 89.60 billion (USD 2.05 billion, 2013 rate). ${ }^{1}$

Emergency Medical Services (EMS) responders, composed of emergency medical technicians, physicians, nurses, and allied health personnel specially trained for emergency response, function as a vital component of disaster medical response (DMR), especially in the aftermath of natural disasters such as that of Typhoon Haiyan, when DMR operations are often affected by poor inter-agency coordination and the lack of essential resources.,3 The preventability of these difficulties has been shown in modeling studies backed by reliable data, ${ }^{4,5,6}$ but a system for assessing the quality of medical response carried out by EMS responders (and thus, the data gathered) has not been validated in the Philippine DMR context.

Disaster medical response planning in the Philippines is governed by the framework of disaster risk reduction and management (DRRM), as mandated by Republic Act 10121 or the Disaster Risk Reduction and Management Act of 2010. DRRM includes these four processes: 1) prevention and mitigation, 2) preparedness, 3) response, and 4) recovery. ${ }^{3}$ Despite defining the scope of areas for disaster management, this framework does not provide for a validated, standardized assessment of the quality of DMR, which can guide subsequent planning for disasters.

Recognizing this lack of standard assessment, European disaster researchers met in Italy, Belgium, and ultimately in Utstein, Norway in 2010 to formulate a template for documenting aspects of DMR that will help improve subsequent planning and response. These gatherings produced the Utstein-style Template for Uniform Data Reporting of Acute Medical Response in Disasters, which assessed 15 disaster-related factors, among them medical communications and information management (nested under disaster medical operations coordination), which is the focus of this article. Specifically, data elements that this template recommends for inclusion in the documentation of DMR include the following:

1. population demographics: tallies collected according to sex and age, local pre-disaster health profile, and documentation of vulnerable groups in the area,

2. health care system capacities: documentation of the local dispatch system, EMS system, and the availability of healthcare facilities, and
3. DMR system: description of the organizational structure, criteria for activation, medical operations plan, concept of operations and medical operations management. ${ }^{3}$

Because DMR planning should be guided by needs that are encountered in the field, this study aimed to document medical needs encountered by Filipino EMS groups in areas that were affected by Typhoon Haiyan, with the intention to inform policy decisions on allocating health resources in responding to disasters of similar magnitude. This study also aimed to identify underlying reasons for difficulties in documenting and responding to these medical needs. In this article, we use "medical needs" to encompass these three aspects that were routinely documented by the study participants during the DMR: triage classification, determination of patient diagnoses, and case management.

\section{METHODS}

\section{Selection of participants and survey}

Research participants were selected from an initial list of 300 Philippine-based EMS responders who were deployed to areas affected by Typhoon Haiyan. This list was supplied by groups vetted by members of the Philippine College of Emergency Medicine (PCEM) and associated EMS organizations. People in the list were invited to complete a study survey, which included items on DMR coordination and medical needs encountered, based on the Utstein-style template. Specifically, the items included in the survey were the following:

1. area of deployment,

2. tally of casualties seen, categorized under either primary cause (i.e. death directly due to the effects of the typhoon) or secondary cause (i.e. death due to a preexisting comorbidity that worsened after the onset of the typhoon),

3. tally of cases according to triage classification. Patients were triaged at the scene under the four categories of the Simple Triage and Rapid Treatment System: T1 (Red/ Immediate), T2 (Yellow/Delayed), T3 (Green/Minimal), and T4 (Black/Blue/Expectant/Dead),

4. tally of cases seen, grouped according to sex and prespecified age ranges, and

5. classification of cases whether these required hospitalization or outpatient management.

In keeping with ethical standards, recruitment was done on an individual voluntary basis and not through the EMS organizations to avoid any possibility that the survey might be misconstrued as a performance evaluation. ${ }^{7}$ To promote participation, potential respondents were informed of the study objectives and the intention to generate policy recommendations to guide future disaster response operations.

Survey responses were sent either via electronic means (i.e. online survey platform) or postal courier. A total of 
52 responses were received, in which 36 of these responses provided data on patient demographic data; only 23 responses included data on triage classification or case management of patients seen. Twelve responses out of the above-mentioned 36 responses were found to be redundant and hence were excluded. The same was also done for five redundant responses out of the 23 that included data on triage classification or case management. (Figure 1).

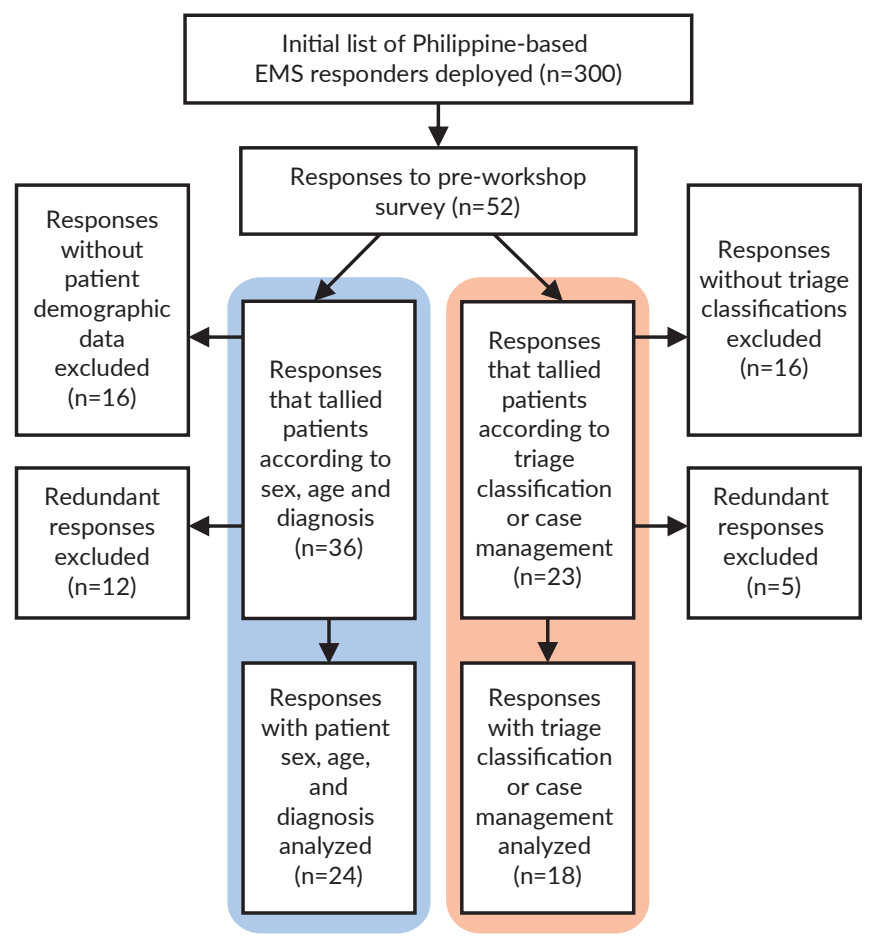

Figure 1. Flow diagram of responses received.

\section{Focus group discussion}

A focus group discussion (FGD) was held in the city of Manila, Philippines in March 2014 to validate survey responses and generate policy recommendations and contextual data related to various concerns that emerged during the DMR. Participants were grouped according to their period of deployment in the Typhoon Haiyan response: 1) responders already in the area during landfall or within the week, 2) responders arriving after one week, 3) responders arriving after two weeks, and 4) responders arriving after three weeks and beyond, to consider these different response activities that correspond with each of the previously mentioned phases of post-disaster response: 1) "saving as much lives as possible," 2) disaster prevention and control, and, in the case of teams arriving beyond three weeks, 3 ) "building back better". ${ }^{8}$

The nominal group technique for consensus building was used for facilitating the subsequent FGDs, which were done simultaneously among the four groups to gather inputs related to the medical needs encountered, and the difficulties experienced. In carrying out this method, a facilitator asked a question on a DMR-related concern derived from the Utstein-style template. Specifically, the FGD asked questions on local health care system capacities, as well as points of improvement and policy recommendations for improving the DMR system. Participants were given a piece of paper to write their response, and affixed their answers on the board. The facilitator grouped similar answers together, and discussed emerging themes. Afterwards, participants were given five (5) dots, which they either affixed on one theme they strongly agreed with, or divided among multiple themes depending on their level of preference. Themes garnering the greatest number of dots were referred to the other groups, whereupon a similar process was done, but now with similar themes across groups clustered together. Finally, themes with the greatest number of dots were chosen as the consensus input of all participants. ${ }^{9}$

In addition to the survey respondents, key persons from the following Philippine agencies and organizations who also participated in the DMR were invited to participate in the focus group discussions: Department of Health, National Disaster Risk Reduction and Management Council (NDRRMC), Metropolitan Manila Development Authority (MMDA), Department of the Interior and Local Government (DILG), Ugnayan ng Pahinungod (a group of volunteers affiliated with the University of the Philippines Manila) and others. Their participation was only limited to policy recommendations in response to inputs generated by the FGDs.

\section{Data collection and analysis}

The Statistical Package for the Social Sciences (SPSS) version 21 and Microsoft Excel (Office 365) were used for computing tallies and generating descriptive statistics.

\section{RESULTS}

Because of the diversity in data recording procedures among the participants' organizations, the tally of patients categorized according to sex, age, and diagnosis (total: 41,202, Table 1) had a discrepancy with tallies according to triage classification, which itself had discrepancies in how the patient information system recorded sex and age group (total according to sex: 19,193; total according to age group: 19,171, see Tables 2 and 3). There was also a discrepancy with tallies according to primary and secondary cause, as well as case management (total according to sex: 27,523; total according to age group: 27,522, see Tables 4 to 6), despite the best effort of the authors in tracking the discrepancy in reported data. Additionally, though all patient tallies included sex, age, and diagnosis, not all were able to record triage information or how their cases were managed (i.e. whether they were hospitalized or not).

\section{Deployment area}

Most of the study participants were deployed to Leyte island (62\%), which was the area with most casualties and damage reported. There were 7 (13\%) responders who were deployed in Samar island and 7 (13\%) responders who went 
Table 1. Adjusted distribution of cases seen by sex and age groups at the point of consult $(n=41,202)$

\begin{tabular}{|c|c|c|c|c|c|c|c|c|}
\hline \multirow{2}{*}{ Type of Cases Seen } & \multirow{2}{*}{ Total } & \multicolumn{2}{|c|}{ Distribution by Sex } & \multicolumn{5}{|c|}{ Distribution by Age (years) } \\
\hline & & Male & Female & 0 to $<1$ & 1 to $<5$ & 5 to 18 & 19 to 65 & $>65$ \\
\hline \multicolumn{9}{|l|}{ Dead } \\
\hline Dead on arrival & 1,090 & 678 & 412 & 33 & 65 & 142 & 621 & 229 \\
\hline Resuscitations (CPR, defibrillator, etc) but dead outcome & 94 & 59 & 35 & 41 & 16 & 1 & 15 & 21 \\
\hline Total Dead & 1,184 & 737 & 447 & 74 & 81 & 143 & 636 & 250 \\
\hline \multicolumn{9}{|l|}{ Alive } \\
\hline Surgical diagnoses & 13,679 & 7,227 & 6,452 & 767 & 2,369 & 3,529 & 4,404 & 2,610 \\
\hline Minor cuts and bruises & & & & & & & & \\
\hline extremities & 2,999 & 1,493 & 1,506 & 450 & 720 & 870 & 629 & 330 \\
\hline head & 2,631 & 1,372 & 1,259 & 105 & 263 & 579 & 974 & 710 \\
\hline between pelvic and knee area & 2,137 & 935 & 1,202 & 107 & 513 & 705 & 470 & 342 \\
\hline between shoulder and hip area & 1,728 & 976 & 752 & 52 & 294 & 449 & 622 & 311 \\
\hline \multicolumn{9}{|l|}{ Major trauma } \\
\hline amputations of extremities & 1,619 & 1,084 & 535 & 16 & 162 & 178 & 648 & 615 \\
\hline head & 1,292 & 665 & 627 & 13 & 245 & 362 & 620 & 52 \\
\hline between pelvic and knee area & 794 & 455 & 339 & 24 & 143 & 262 & 230 & 135 \\
\hline between shoulder and hip area & 479 & 247 & 232 & 0 & 29 & 124 & 211 & 115 \\
\hline Post-traumatic depression & 2,663 & 2,062 & 601 & 0 & 479 & 852 & 1,172 & 160 \\
\hline Pulmonary & 2,556 & 946 & 1,610 & 358 & 690 & 460 & 613 & 435 \\
\hline Infectious & 2,210 & 1,101 & 1,109 & 420 & 619 & 464 & 243 & 464 \\
\hline Cardiovascular & 1,893 & 687 & 1,206 & 303 & 303 & 454 & 530 & 303 \\
\hline Gastrointestinal & 1,848 & 647 & 1,201 & 462 & 536 & 444 & 240 & 166 \\
\hline Musculoskeletal & 1,095 & 585 & 510 & 11 & 66 & 164 & 854 & 0 \\
\hline Drowning & 322 & 187 & 135 & 151 & 103 & 29 & 16 & 23 \\
\hline Fever & 34 & 5 & 29 & 2 & 3 & 10 & 18 & 1 \\
\hline Deliveries & 28 & 0 & 28 & 0 & 0 & 0 & 28 & 0 \\
\hline Allergy & 11 & 6 & 5 & 1 & 2 & 3 & 5 & 0 \\
\hline Total Alive & 40,018 & 20,680 & 19,338 & 3,242 & 7,539 & 9,938 & 12,527 & 6,772 \\
\hline GRAND TOTAL (All cases seen) & 41,202 & 21,417 & 19,785 & 3,316 & 7,620 & 10,081 & 13,163 & 7,022 \\
\hline
\end{tabular}

to Panay island. The rest of the responders were deployed in Villamor Air Base in Pasay City (8\%) and in Cebu (4\%), which was a hub for airlifted families and patients, and was the receiving end of communication efforts from the affected areas. (Figure 2).

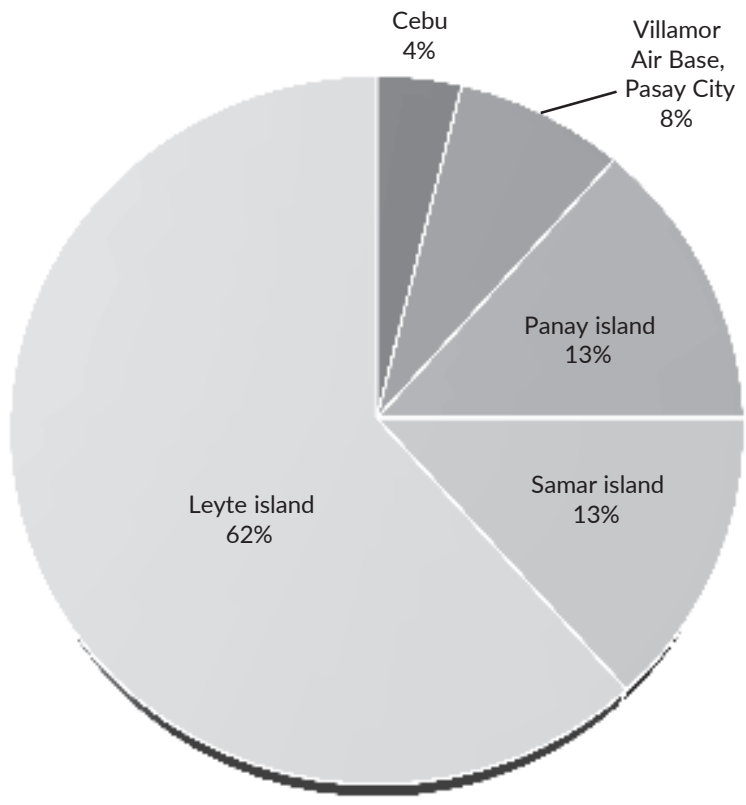

Figure 2. Respondents' location of deployment ( $n=52)$.
Patients categorized according to sex, age, and diagnosis

As shown in Table 1, among responses with reported sex, age, and diagnoses, 41,202 cases were tallied, of which 40,018 (97.13\%) were seen alive, 1,090 (2.65\%) were dead on arrival, and $94(0.23 \%)$ died despite resuscitation efforts. Among patients seen alive, 20,680 (51.67\%) were male and $19,338(48.32 \%)$ were female.

For purposes of analysis we used these age groups: 1 ) infants 0 to less than 1 year old, 2) children aged 1 to less than 5 years, 3 ) children aged 5 to 18 years, 4 ) adults aged 19 to 65 years, and 5) elderly aged more than 65 years. The choice of age grouping scheme was made on the bases of health program implementation in the Philippine setting, with special programs allocated to infants, young children, and the elderly. However, due to data recording errors or discrepancies

Table 2. Adjusted distribution of cases according to sex and triage category $(n=19,193)$

\begin{tabular}{lrr}
\multirow{2}{*}{ Triage Category } & \multicolumn{2}{c}{ Distribution by Sex } \\
\cline { 2 - 3 } & Male & Female \\
\hline T1 cases (Red/emergent) & 1,044 & 1,028 \\
T2 cases (Yellow/urgent) & 2,156 & 1,678 \\
T3 cases (Green/non-urgent) & 7,382 & 4,673 \\
T4 cases (Black/ Blue/expectant) & 520 & $\mathbf{7 1 2}$ \\
\hline TOTAL & $\mathbf{1 1 , 1 0 2}$ & $\mathbf{8 , 0 9 1}$ \\
\hline
\end{tabular}


Table 3. Adjusted distribution of cases according to age group and triage category $(n=19,171)$

\begin{tabular}{lrrrrr}
\multicolumn{1}{c}{ Triage Category } & \multicolumn{5}{c}{ Distribution by Age } \\
\cline { 2 - 6 } & $\mathbf{0}$ to $<\mathbf{1}$ yo & $\mathbf{1}$ to $<\mathbf{5}$ yo & $\mathbf{5}$ to $\mathbf{1 8}$ yo & $\mathbf{1 9}$ to $\mathbf{6 5}$ yo & $>\mathbf{6 5}$ yo \\
T1 cases (Red/emergent) & 826 & 208 & 418 & 245 & 416 \\
T2 cases (Yellow/urgent) & 1,066 & 378 & 422 & 1,187 & 762 \\
T3 cases (Green/non-urgent) & 2,056 & 3,152 & 2,378 & 3,439 & 1,241 \\
T4 cases (Black/ Blue/expectant) & 0 & 0 & 125 & 0 \\
\hline TOTAL & 3,948 & 3,738 & 3,343 & 5,723 & 2,419 \\
\hline
\end{tabular}

Table 4. Adjusted distribution of cases according to primary and secondary cause $(n=27,523)$

\begin{tabular}{|c|c|c|c|c|c|c|c|}
\hline \multirow{2}{*}{ Types of cases seen } & \multicolumn{2}{|c|}{ Distribution by Sex } & \multicolumn{5}{|c|}{ Distribution by Age (years) } \\
\hline & Male & Female & 0 to $<1$ & 1 to $<5$ & 5 to 18 & 19 to 65 & $>65$ \\
\hline Primary cause (drowning, asphyxiation, hypothermia, trauma) & 8,151 & 7,034 & 992 & 2,553 & 3,701 & 5,056 & 2,883 \\
\hline $\begin{array}{l}\text { Secondary cause (hypertension, diabetes, congenial and chronic } \\
\text { diseases, or other injuries sustained after the typhoon, etc.) }\end{array}$ & 6,039 & 6,299 & 1,557 & 2,698 & 2,851 & 3,703 & 1,529 \\
\hline TOTAL & 14,190 & 13,333 & 2,549 & 5,251 & 6,552 & 8,759 & 4,412 \\
\hline
\end{tabular}

Table 5. Adjusted distribution of cases according to sex, and hospitalized and non-hospitalized cases ( $n=27,523$ )

\begin{tabular}{lcr}
\multirow{2}{*}{ Types of cases seen } & \multicolumn{2}{c}{ Distribution by Sex } \\
\cline { 2 - 3 } Hospitalized cases & Male & Female \\
Non-hospitalized cases & 2,993 & 2,880 \\
\hline TOTAL & 11,197 & 10,453 \\
\hline
\end{tabular}

in reporting, there were 4,146 patients unaccounted for in terms of age. We thus adjusted the total tally based on the assumption that the aggregated age distribution of patients with available age data can approximate the percentage distribution of the overall population.

Furthermore, 13,679 (34.18\%) were cases requiring surgical management, of which 9,495 (69.41\%) involved minor cuts and bruises on the extremities, the head, and the pelvic, knee, shoulder, and hip areas. The remainder of these cases $(4,184$ or $30.59 \%)$ involved major trauma of the aforementioned areas or traumatic amputation of extremities. (Table 1).

Non-surgical diagnoses seen at the point of consult included cases of post-traumatic stress or depression $(2,663$ or $6.65 \%)$, pulmonary cases $(2,556$ or $6.39 \%)$, and infectious cases $(2,210$ or $5.52 \%)$, as well as cardiovascular, gastrointestinal, and musculoskeletal cases, which accounted for less than $5 \%$ of cases. Accounting for less than $1 \%$ was drowning, fever of unknown origin, maternal deliveries, and allergies. An interesting finding in these tallies was the prevalence of post-traumatic depression more among male patients $(2,062$ of 2,663 cases, or $77.43 \%)$, which deserves further study. (Table 1).

\section{Patients categorized according to triage classification}

Among responses with reported triage classification, a total of 19,193 patients were tallied, of which 11,102 (57.84\%) were male patients while 8,091 (42.16\%) were female (Table 2).

Notwithstanding the discrepancies between data based on sex (Table 2) and age group (Table 3), patients triaged as $\mathrm{T} 1$ were $11 \%$ of all the cases seen. Most of these patients (40\%) belonged to the 0 to $<1$ age group. Meanwhile, T2 cases comprised $20 \%$ of the cases seen, wherein $31 \%$ were from the 19 to 65 age group, and $28 \%$ were from the 0 to $<1$ age group. Moreover, T3 comprised of $63 \%$ of the cases, $29 \%$ of which belonged to the 19 to 65 age group. Finally, those classified as expectant or dead (T4) comprised $6 \%$ of the cases, of which $69 \%$ were from the 19 to 65 age group and $10 \%$ were from the 5 to 18 age group.

\section{Patients with reported case management}

Among responses with reported case management, a total of 27,523 patients were tallied, of which 14,190 (51.56\%) were male patients while 13,333 (48.44\%) were female. Out of the total tally, 12,338 patients (45\%) sought consult for conditions attributable to secondary causes, such as hypertension, diabetes, congenital conditions, minor trauma, and other chronic diseases. This was outnumbered by 15,185 or $55 \%$ of the total cases, which resulted from primary causes (drowning, asphyxiation, hypothermia, trauma). Moreover, 5,056 or $33 \%$ of cases attributable to primary causes came from the 19 to 65 age group (Table 4).

Table 6. Adjusted distribution of cases according to age group, and hospitalized and non-hospitalized cases ( $\mathrm{n}=27,522 *$ )

\begin{tabular}{lccccc}
\multicolumn{1}{c}{ Types of cases seen } & \multicolumn{5}{c}{ Distribution by Age Group } \\
\cline { 2 - 6 } & $\mathbf{0}$ to $<1$ yo & 1yo to $<5$ yo & $\mathbf{5}$ to 18 yo & $\mathbf{1 9}$ to $\mathbf{6 5}$ yo & $>\mathbf{6 5}$ yo \\
Hospitalized cases & 463 & 1,655 & 1,564 & 2,170 & 472 \\
Non-hospitalized cases & 2,061 & 3,609 & 4,998 & 6,590 & 3,940 \\
\hline TOTAL & 2,524 & 5,264 & 6,562 & 8,760 & 4,412 \\
\hline
\end{tabular}

*Author's note: Despite our best effort in tracking the discrepancy, there is one patient unaccounted for in this tally from the one in Table 5. 
Furthermore, 5,873 (21\%) were hospitalized and 21,650 (79\%) were managed on an outpatient basis. There was almost an equal ratio between both sexes for those hospitalized wherein 2,993 (51\%) were male and 2,880 (49\%) were female. Most (34\%) of the hospitalized cases belonged to the 19 to 65 age group. Majority of those who were seen and not hospitalized were male 11,197 (51.72\%) and belonged to the 19 to 65 age group, 6,590 (30\%). (Tables 5 and 6).

\section{Focus group discussion}

Focusing on communication and information management, participants agreed that there was lack of central coordination and there was absence of a unified communication or information management system among responders, thereby impeding respondents from reporting scarcity of resources, manpower, and supplies, as well as proper endorsement between responders and receiving hospitals. They also agreed that addressing these concerns requires that DMR groups use a standard reporting form and other data gathering tools provided by the Department of Health, designate an information office tasked with ensuring data quality, and attend cluster meetings with other DMR-oriented organizations. Additionally, the participants emphasized the significant role of the Department of Health - Health Emergency Management Bureau in swiftly coordinating the DMR efforts carried out by various EMS groups and other volunteer organizations, since deployment often occurs due to urgency perceived by the deploying organization or individual volunteers. Notably, the "spirit of volunteerism" was noted as a significant motivating factor to participate in the response. Thus, to facilitate multiagency coordination with the EMS sector, the participants also recommended the creation of a national registry for emergency medical responders.

\section{DISCUSSION}

Assessing the tallies gathered by the study at face value, and assuming that all assessments were done correctly according to triaging guidelines, we find that most patients seen by study participants were triaged as T3, a classification that requires minimal direct patient intervention, but also underlines the need for proper assessment by adequately trained and equipped responders. ${ }^{10-11}$ Moreover, an overwhelming majority of the patients tallied by the survey respondents had complaints that were managed surgically. Also, most patients sought consult for complaints attributable to secondary causes, highlighting an already demonstrated importance of providing continuity of care for noncommunicable diseases, mental health disorders, and other chronic conditions, as was seen in events of similar magnitude like Hurricane Ike in the United States (2008) and the seasonal cyclones in Japan, United States, and the Philippines, as well as India, Bangladesh, and other tropical countries. ${ }^{12-14}$
However, notwithstanding how these data pinpoint to the need to consider the level of training of deployed personnel and the ability to address specific diagnoses or provide continuity of care, a more significant issue is the lack of data gathering standards in disaster-affected areas, as demonstrated by the insufficient triage classification data and the varying systems of documentation among participants. It is a relatively common phenomenon that has been attributed to these factors: the environmental nature of disasters, personal safety, challenges related to communication (specially with other disaster response organizations), and socioeconomic, political, and cultural considerations. ${ }^{15}$ These factors thus demonstrate the importance of effective communication and multi-agency coordination and collaboration in ensuring accuracy of DMR data gathering.

Since the establishment of DMR is usually chaotic within affected agencies, disaster planning must include communication protocols, contingency plans, and organizational charts that will help identify offices that are responsible for each of the DRRM phases (i.e. prevention and mitigation, preparedness, response, and recovery)., $3,8,16,18$ Templates for reporting must also be agreed with stakeholders involved in DMR, but these should be harmonized with a national standard, to facilitate equitable allocation of resources, especially in disasters that affect several areas.

However, setting a national standard is often fraught with organizational and political challenges, such as levelling of conflicting interests, and lengthy bureaucratic and consultative processes, which are interventions at the policymaker level, access to which the EMS sector has only been provided marginally in the Philippine context. ${ }^{16-17}$ For instance, there is no explicit mention of the EMS sector among organizations represented in the NDRRMC and the local councils. ${ }^{18}$ Because the EMS sector encompasses organizations with links to both government and private agencies and a scope that ranges from national to local levels, we believe it greatly behooves the NDRRMC to give this sector a greater role in DRRM policy development because of the wealth of experience and expertise of its members in DMR. This is to help avoid the lack of clarity in the roles to be assumed by private sector organizations during disaster response, and the "top-heaviness" of disaster response planning, made apparent by the difficulty in scaling operations to actual service delivery in the affected areas. ${ }^{19}$

\section{Limitations}

This study is limited by its relatively small sample size, which affects the generalizability of the results. Additionally, the study was unable to document the level of satisfaction of responders in the way their intended medical management was carried out, which would produce a more compelling argument for allocating health resources appropriately, as this would require substantial additions to the survey template and a separate FGD for each of the diagnostic categories. Moreover, the nominal group technique, which 
is inherently a tool for consensus building, did not intend to relate medical needs and other related issues with period of deployment, focusing instead on priority actions in response to the needs identified and agreed on by all participants, regardless of their period of deployment. There were also no representatives from international organizations such as the agencies of the United Nations, which would have generated inputs on the extent of difficulty in scaling national-level disaster planning to implement health service delivery in affected areas. Investigating relationships between all the aforementioned variables would additionally assist in planning DMR activities by allowing the consideration of geographical, social, political, and other epidemiological considerations, and thus, deserves further study.

\section{CONCLUSION AND RECOMMENDATIONS}

Reliable information is essential for effective and equitable allocation of resources to address medical needs encountered in a disaster of such magnitude as that of Typhoon Haiyan. Thus, the difficulty of documenting cases seen in the context of DMR carried out by EMS responders pinpoints aspects of health services delivery, multi-agency coordination, and information management that require further strengthening. These are concerns that can be addressed sustainably by policy interventions, which necessitate significant political capital in the Philippine setting. As such, stakeholders from the EMS sector should be represented in DMR planning, a role which will be sustainably ensured by legislation.

\section{Acknowledgments}

The authors would like to thank Dr Michel Debacker and Dr Marcel van der Auwera of the Research Group on Emergency and Disaster Medicine, Vrije Universiteit Brussels, Belgium; Dr Merritt D Schreiber of the Center for Disaster Medical Sciences, University of California, Irvine, USA; and Dr. Erin Hughey of the Pacific Disaster Center for their technical inputs, and for providing permission to use data gathering templates and psychosocial analysis tools that they developed. We gratefully acknowledge the cooperation of all study participants, as well as the service carried out by all frontline health workers during the DMR. We would also like to thank Ms. Regina Abola for her assistance in the preparation of this manuscript, and the reviewers for their helpful comments.

\section{Ethical clearance}

The UP Manila Research Ethics Board gave ethical clearance for this study under protocol number 2014-087-01. Participants were given compensation for work-hours spent on participating in the study, travel expenses incurred, and accommodation for those who came from the provinces.

\section{Statement of Authorship}

All authors approved the final version submitted.

\section{Author Disclosure}

All authors declared no conflict of interest.

\section{Funding Source}

This paper was funded by Pacific Disaster Center, Hawaii, USA.

\section{REFERENCES}

1. National Disaster Risk Reduction and Management Council. Final report re effects of Typhoon "Yolanda" (Haiyan). 2009 November 6-9. Quezon City, Philippines. http://ndrrmc.gov. ph/attachments/article/ 1329/FINAL_REPORT_re_Effects_of_ Typhoon_YOLANDA_(HAIYAN) _06-09NOV2013.pdf.

2. Catlett CL, Jenkins JL, Millin MG. Role of emergency medical services in disaster response: resource document for the National Association of EMS Physicians position statement. Prehosp Emerg Care. 2011; 15(3): 420-5. doi: 10.3109/10903127.2011.561401. Epub 2011 Apr 11.

3. Debacker M, Hubloue I, Dhondt E, et al. Utstein-style template for uniform data reporting of acute medical response in disasters. PLoS Curr. 2012; 4:e4f6cf3e8df15a. doi: 10.1371/4f6c3e8df15.

4. Potter MA, Miner KR, Barnett DJ, et al. The evidence base for effectiveness of preparedness training: a retrospective analysis. Public Health Rep. 2010; 125 Suppl 5:15-23.

5. Barri Khojasteh S, Macit I. A stochastic programming model for decision-making concerning medical supply location and allocation in disaster management. Disaster Med Public Health Prep. 2017; 5:1-9. doi: 10.1017/dmp.2017.9.

6. Gowing JR, Walker KN, Elmer SL, Cummings EA. Disaster preparedness among health professionals and support staff: what is effective? An integrative literature review. Prehosp Disaster Med. 2017; 32(3):321-8. doi: 10.1017/S1049023X1700019X.

7. Council for International Organizations of Medical Sciences. Guideline 13, Research involving vulnerable persons. International Ethical Guidelines for Epidemiological Studies. In: Compilation of selected guidelines for health research ethics and good clinical practice. National Institutes of Health, University of the Philippines Manila, Manila, Philippines. 2014.

8. Go M, Lopez J. The Philippine disaster management system. Graduate School of Public and Development Management, Development Academy of the Philippines, Pasig City, Philippines. 2016. Unpublished.

9. Kline T. Assessing the utility of the nominal group technique as a consensus-building tool in extension-led avian influenza response planning. J Extension. 2013; 51(5).

10. Ramos R, De los Reyes V, Sucaldito M, Tayag E. Rapid health assessments of evacuation centres in areas affected by Typhoon Haiyan. Western Pac Surveill Response J. 2015; 6 (Suppl 1):39-43. doi: 10.5365/WPSAR.2015.6.2.HYN_003.

11. Nomura S, Blangiardo M, Tsubokura M, Ozaki A, Morita $\mathrm{T}$, Hodgson S. Postnuclear disaster evacuation and chronic health in adults in Fukushima, Japan: a long-term retrospective analysis. BMJ Open. 2016; 6(2):e010080. doi: 10.1136/ bmjopen-2015-010080.

12. Ryan B, Franklin RC, Burkle FM Jr, et al. Identifying and describing the impact of cyclone, storm and flood related disasters on treatment management, care and exacerbations of non-communicable diseases and the implications for public health. PLoS Curr. 2015; 7. doi:10.1371/ currents.dis.62e9286d15 2de04799644dcca47d9288.

13. Pietrzak RH, Southwick SM, Tracy M, Galea S, Norris FH. Posttraumatic stress disorder, depression, and perceived needs for psychological care in older persons affected by Hurricane Ike. J Affect Disord. 2012; 138(1-2):96-103. doi: 10.1016/j. jad.2011.12.018. Epub 2012 Jan 28. 
14. Nahar N, Blomstedt $\mathrm{Y}, \mathrm{Wu}$ B, Kandarina I, Trisnantoro L, Kinsman J. Increasing the provision of mental health care for vulnerable, disaster-affected people in Bangladesh. BMC Public Health. 2014; 14:708. doi: 10.1186/1471-2458-14-708.

15. Morton M, Levy JL. Challenges in disaster data collection during recent disasters. Prehosp Disaster Med. 2011; 26(3):196-201. doi: 10.1017/S1049023X11006339.

16. Jha A, Basu R, Basu A. Studying policy changes in disaster management in India: A tale of two cyclones. Disaster Med Public Health Prep. 2016; 10(1):42-6. doi: 10.1017/dmp.2015.116.

17. Alcayna T, Bollettino V, Dy P, Vinck P. Resilience and disaster trends in the Philippines: opportunities for national and local capacity building. PLoS Curr. 2016; 8. doi:10.1371/currents.dis. 4a0bc960866e53bd6357ac135d740846.
18. Republic Act 10121. An act strengthening the Philippine disaster risk reduction and management system, providing for the National Disaster Risk Reduction and Management Framework and institutionalizing the National Disaster Risk Reduction and Management Plan, appropriating funds therefor and for other purposes. Congress of the Philippines, Metro Manila, Philippines. 2010.

19. Tiller S. The 'new humanitarian landscape.' Case study: Philippines Typhoon Haiyan response. Doctors Without Borders (Medecins sans frontieres), pp. 5, 16. 2014 April. https:// www.msf.org.uk/sites/uk/files/humanitarian_aid_landscape_-philippines.pdf.

The Acta Medica Philippina is now accepting limited advertising for its front and back cover (colored), as well as for available spaces in some of its pages, as appropriate. For inquiries and submission of proposals, please e-mail us at editor@actamedicaphilippina.org 